\title{
Article
}

\section{Diabetic Retinopathy Diagnostics from Retinal Images based on Deep Convolutional Networks}

\author{
G. Arun Sampaul Thomas' ${ }^{1}$, Y. Harold Robinson'2, E. Golden Julie ${ }^{3}$, Vimal Shanmuganathan ${ }^{4}$, \\ Yunyoung Nam ${ }^{5}$, Seungmin Rho $^{6^{*}}$ \\ 1 CSE Department, J.B. Institute of Engineering and Technology, Hyderabad, India. \\ Email:arunthomas.cse@jbiet.edu.in \\ 2 School of Information Technology and Engineering, Vellore Institute of Technology, Vellore, India. \\ Email:yhrobinphd@gmail.com \\ 3 Department of Computer Science and Engineering, Anna University Regional Campus, Tirunelveli, India. \\ Email:goldenjuliephd@gmail.com \\ 4 Department of Information Technology, National Engineering College, Kovilpatti, India, \\ Email:svimalphd@gmail.com \\ 5 Department of Computer Science and Engineering, Soonchunhyang University, Asan 31538, Korea, \\ Email: ynam@sch.ac.kr \\ 6 Department of Software, Sejong University, Seoul, 05006, Korea, Email: smrho@sejong.ac.kr \\ * Correspondence: smrho@sejong.edu (S.R.)
}

\begin{abstract}
Retinopathy is a human eye disease that causes changes in retinal blood vessels that leads to bleed, leak fluid and vision impairment. Symptoms of retinopathy are blurred vision, changes in color perception, red spots, and eye pain. In this paper, a new methodology based on Convolutional Neural Networks (CNN) is developed and proposed to diagnose and give a decision about the presence of retinopathy. The CNN model is trained by different images of eyes that have retinopathy and those which do not have retinopathy. The performance of the proposed model is compared with the related methods of DREAM, KNN, GD-CNN and SVM. Experimental results show that the proposed $\mathrm{CNN}$ performs better.
\end{abstract}

Keywords: Convolutional Neural Networks; Dental Diagnosis; Image Recognition; Diabetic Retinopathy detection

\section{Introduction}

Diabetic retinopathy conjointly is called diabetic diseases once the injury happens to the tissue layer because of polygenic disease [1]. It is a general illness that imitates up to $80 \%$ of all patients [2]. The analysis showed that a minimum of $90 \%$ of those latest cases might well be abridged if there have been correct and alert healing with the observation of the eyes [3]. An aged and individual has a polygenic disease with the probabilities of developing diabetic retinopathy. A huge quantity of adults having diabetes in the world is approximated to 366 million in 2011 and 552 million by 2030 [4]. The numeral figure of individuals with this kind a pair of polygenic disease is mounting in each country where $80 \%$ of individuals with the polygenic disease [5]. The current studies demonstrate an increasing dominance in rural areas [6-8]. Several patients do not have any clinically visible Diabetic Retinopathy (DR) premature once diagnosing; however, there are recognized structural variations in the retina with the induced amount of retinal bloodstream, augmented leukocyte devotion, clotting of vault membranes [10]. The hastiest clinically evident period of DR is non-proliferative diabetic retinopathy (NPDR) pigeonholed by the enlargement of microaneurysms [11].

The illness will reach restrained NPDR anywhere supplementary DR lesions grow, together with venous caliber variations and intra-retinal microvascular abnormalities [12]. The sternness and 
amount of those lesions have ensued in brutal NPDR, and retinal blood deliver progresses progressively more negotiation [13]. As a corollary, the non-perfused parts of the retina direct signals inspiring fresh blood vessel enlargement, according to proliferative diabetic retinopathy (PDR) [14]. The fresh blood vessels are square measure irregular, quickly frequently causing harsh visual loss [15]. Diabetic Macular Edema (DME) [15] ensues when there is swelling with serious of the retina owing to the dripping of fluid from blood vessels inside the macula and may appear during any period of DR [16]. The succession from no retinopathy to PDR may obtain this slow rate that supports DR to be acknowledged and indulgenced at a preliminary phase [17]. Expansion and succession of DR are said to stage and management of the polygenic disease. DR in its initial type is usually well but flexible to healing [18]. The Initial healing of Diabetic Retinopathy Study [19] showed the treatment with optical device surgical operation that will quite fraction the danger of embryonic visual failure from PDR [20]. In common, classification is made by extorting the descriptions with the images monitored by recognizing the generated classes according to the input data [21-22]. In categorization, the extorted features enumerated with related to the particular disease [23]. The category-based extortion is used to analyze the particular disease [24].

The proposed method is utilized that the increment of the spatial resolution compared with the methods. It is automatically identified the feature extraction for diabetic retinopathy. The validation and training errors have been identified whenever the total amount of iterations is increased. The Convolutional Neural Networks are the common computer vision methodology with fully connected networks with the dissimilar metrics. The fully connected layers can perform the classification of the real-time images. The proposed method also used to reduce the computational cost in several parameters. The pooling layers are introduced to minimize the coherence within the adjacent layers. The classification has been achieved using the proposed method that utilized the processing power is required to learn the kernel values and reached the target value within the inference. The proposed method also identified the compound patterns in the adjacent Convolutional layers. The patterns are identified while the kernel has 100 percent matching. The feature loss factor is used to increase the label value. The methodology is based on the performance metrics of specificity and sensitivity for identifying glaucoma, our proposed methodology is used to perform the parameters of precision, recall and accuracy that will produce the highest amount of accuracy compared to the previous methodology. The pre-training technique is implemented into the selected local images to produce the result. But in our model, the pre-training technique is performed in more amounts of images that will increase the enhanced performance.

The contribution of the paper is

$\checkmark$ A new model that uses the Convolutional Neural Network (CNN) to increase the accuracy of Retinopathy Diagnosis.

$\checkmark$ The proposed algorithm is constructed to eliminate the feature extraction hurdles and also used to reduce the computational power to gather the highest amount of accuracy.

$\checkmark$ The model is trained by different images of eyes that have retinopathy and those which do not have retinopathy.

$\checkmark$ Experimental results confirmed the advantages of the new model.

\section{Related works}

Several methods are used to implement Diabetic Retinopathy (DR) detection from Retinal images. DR detection with retinal images used Machine Learning (ML) framework classification, which was lacked to define the level of DR identification [25]. The fundus images-based DR detection dealt with spatiotemporal images but took more time for execution [26]. Convolutional Neural Network (CNN) was used to classify the image based on the Random Forest (RF) classifier [27]. An innovative Deep Learning methodology was constructed for microaneurysm recognition [28]. An involuntary classification of blood vessels in fundus images was implemented with the novel technique in [29]. An established technique of diabetic retinopathy identification device was 
constructed with preprocessing [30]. A new methodology was implemented to finalize the identification of diseases [31]. Fundus images are utilized to identify the same [32]. Another methodology was used for diagnostic retinopathy [33]. The method is implemented for the discovery of the exudates in the color unpredictability and dissimilarity retinal images [34]. A lot of other techniques used classification algorithms like KNN [35], Random forest [36], SVM [37], Naïve Bayes [38] which do not best fit for the dataset that encloses images.

The specificity and sensitivity are observed for identifying diabetic retinopathy which is confirmed by the ophthalmologist panel. The large amount of images are validated in the real-time data set, the deep learning based algorithms are very useful to generate the specificity and sensitivity. The algorithm has been evaluated using the numbers within 0 to 1 for identifying the diabetic retinopathy with the probability and classifications. The operating curves have been plotted by contrasting the curve points and threshold value from the development set. The initial operating point is used to identify the specificity and the adjacent point is used to discover the sensitivity. The sensitivity with highest value is the primary element for the screening tool [41].

The Diabetic Retinopathy has been undiagnosed in many occasions that will cause the preventable blindness. To reduce this, the robust diagnostic system has to be developed with automatic Diabetic Retinopathy screening with accurate diagnoses. The deep learning technique based diagnostic tool has been developed with automatic detection from the fundus images. The characteristic curve is the parameter to evaluate the precision and recall of every fundus images from the data set. An artificial intelligent related algorithm is utilized to process the fundus images which are observed from the diabetic patients [42].

The diabetic retinopathy is the measurement within the ocular biometry and refractive error. The population related investigation is normally processed around the diabetic patients. The Early Treatment based study is completed and having the clinically considerable threshold values [43]. The temporal retinal arteries are evaluated using the associations within the optic disc metrics. The curvature from the surface and the point of retinal vessel trunk are the positions to identify the refractive error. The angle is measured according to the images from the dataset. The linear regression with Bayes coefficients are utilized to analyze the covariance parameter within the spherical refractive error and mean variation. The optic nerve head is mainly concentrated on myopic patients. The optical coherence tomography has been implemented to the images for depth identification of retina [44].

The relationship within the Spherical Equivalent and blood vessel along with the functional diagnostic component like glaucoma is used to discover the parameter for the development of optical coherence tomography. The visual fields of all the patients are analyzed to discover the fixation loss using OCT scan. The thickness of the retinal images that matches the relevant fundus images then the particular centre is excluded from the preprocessing state. The linear regression based angular position is identified with the evidence for vein positions [45]. The glaucomatous optic neuropathy has been detected using an advanced deep learning system with improved specificity and sensitivity within the retinal fundus images. The various validated datasets were allowed to access the dissimilar image quality related on fundus images which are collected from the various websites. The population related evaluation has been performed to discover the diabetic retinopathy diagnosis [46].

\section{Proposed Work}

\subsection{Main ideas}

The main issue with the related methodologies is the pre-processing of the pattered images to improve the features. Enhancement of the images for extracting the features will be inaccurate to import the high value of resolution. Diabetic Retinopathy is a human eye disease that will be automatically detected using the efficient methodology. The Image resizing metric is one of the main issues for training images because of minimized computational power. The feature loss factor is in increasing value whenever the labels of the image may vary. To address this kind of problem, 
Convolutional Neural Network (CNN) is used with the dissimilar capacities in view of predicting and training the images. Every image with the statistical information is trained with the neural models that can be capable of justifying the dissimilar usage of statistical methodology. The proposed Convolutional Neural Network with fully connected layers is utilized to classify the images from the American Sign Language data set which contains more than 45000 images. The images can be segregated into Training set and testing set. The proposed methodology is classified with the output classes and pixel values. The entire network is trained with Backpropagation methodology. The ReLU is constructed with feature maps and bias values. The optimized weight values are computed for generating the best methodology.

\subsection{Convolution Neural Networks}

The model is built using Keras and Tensor Flow as the backend. Tensor Flow is chosen as the backend due to better performance over Theano, and the ability to visualize the neural network using Tensor Board. For forecasting two categories, Eye Net utilizes three Convolutional layers, individually having a depth of 32. Max Pooling layer is implied after all three Convolutional layers with size $(2,2)$. After assembling, the data are nourished within a particularly dense layer of size 128, and lastly to the output layer, containing two Softmax nodes.

A Convolutional Neural Network is a feed-forward artificial neural network that is connected where the pattern within its neurons is developed using the visual cortex so that individual neurons are organized with the overlapping regions within the visual field. It uses a complicated structure with the stacked layers to be adapted to identify the images. The constructed framework is used for multi-class classification that every feature is identified within the image. Fifteen layers have the weights whose 13 layers are Convolutional and the rests are entirely linked. Our proposed model enlarges the logistic regression value for the multinomial element. The Max-pool layer is framed using 3 Convolutional layers that need to improve accuracy. Each Convolutional layer is framed using the ReLU layer to increase the training space.

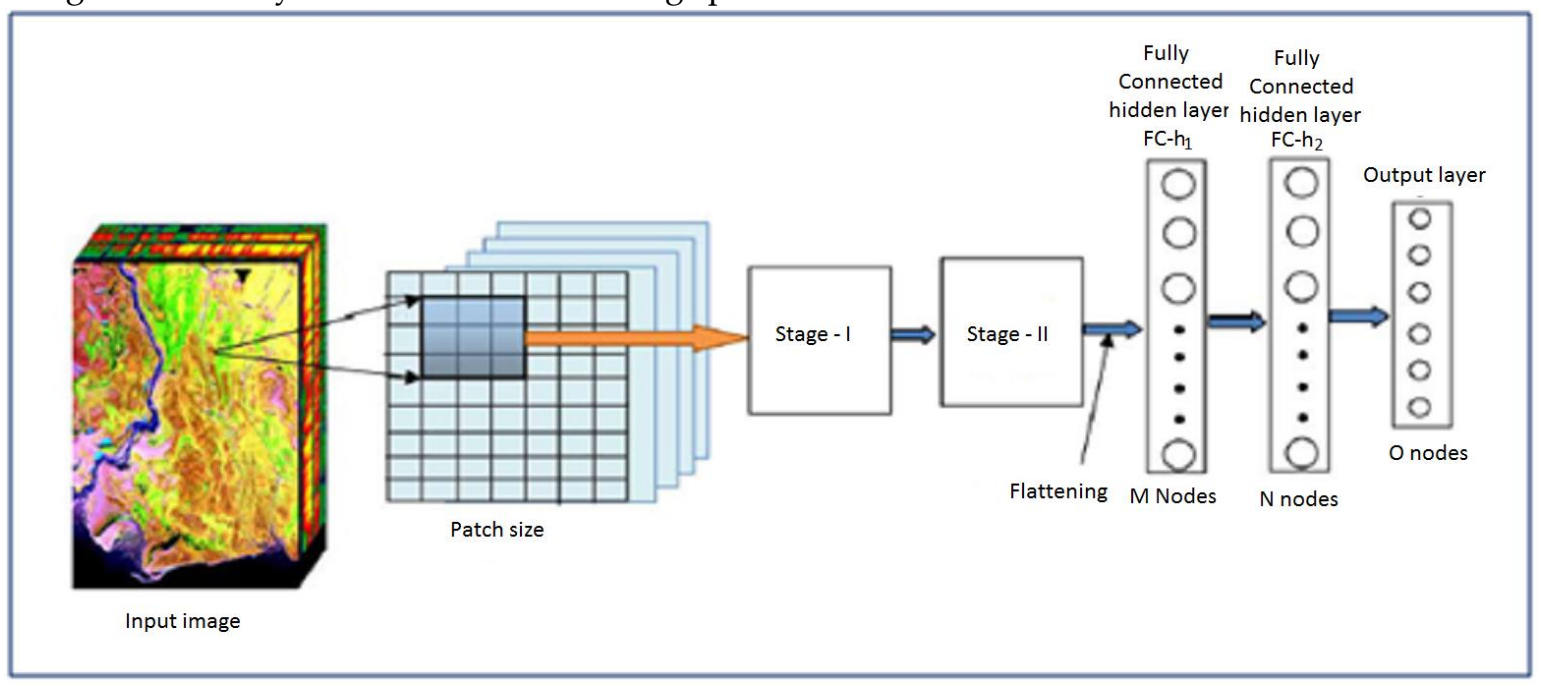

Figure 1. CNN Architecture. 


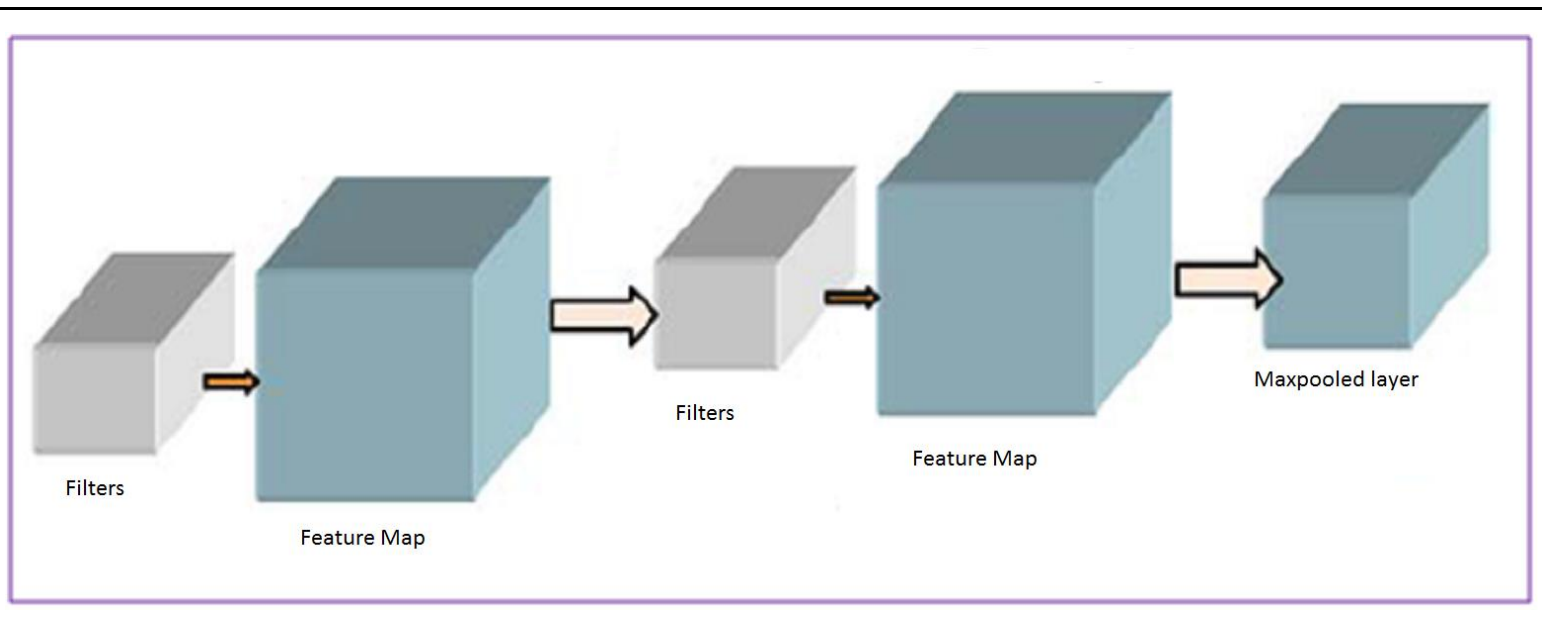

Figure 2. Max pooling stages.

The Convolutional Neural Networks mechanism is demonstrated in Figures $\mathbf{1}$ and 2. The CNN is the combination of the Convolutional layer and spatially related features to advance the classification performance. The architecture design contains the Fully Connected Layers. The output of this model is classified using the label classes Output ${ }_{i}$ for particular pixels Pixel $_{i}$. The complete network is trained using the backpropagation algorithm with error handling methods. The filter for controlling Convolution layers and the weight for the Fully Connected Layers are completed based on the errors. The extraction is done using the Pixel $_{i}$ based on the image sizes with spectral features extracted from the images. The adjacent pixel contains the patch and the connecting window for the adjacent patches. Finally, the patch contains a pixel to concentrate on the Maxpool layers. The human interaction system contains the complex values that integrate outputs for adjacent values. The complex values are invariant to the features and shared weights. The connection based on the correlation values is integrated with the generalization of the methodology. The ReLU formation for the activation function is denoted as Function $_{i}$ having the total amount of feature maps of the size $\operatorname{Re}_{p} \times C_{p}$. The initial convolution layer is demonstrated in Eq. (1)

$$
A c t_{i}^{l}=\sum_{j} f u n\left(w t_{i, j}^{l} * A c t_{i}^{l-1}+\operatorname{Bias}_{i}^{l}\right)
$$

The feature map for the Convolutional Layer is computed using Eq. (2).

$$
\text { Feature }_{\text {map }}(i)=\operatorname{fun}\left(\text { Act }_{i}^{l}\right)=\max \left(0, \operatorname{Act}_{i}^{l}\right)
$$

The input image for classifying the computational resources is achieved using the zero-padding technique for filter value and kernel values. The computational speed and the total amount of class labels are essential for computing the Feature map for the input image. The filters for every Convolutional layer use depth and common edges for huge amount of feature maps for reducing the computational time and storage space. Therefore, the parameters are used to achieve stability for a particular problem. The pixels which are used to balance the operation of the input image are computed using the parameter stride. The filter shifts one pixel at a period whenever the single stride. Padding with zeros in the spatial based feature map is used in the input image.

In the initial stage, the number of features captured from the $1^{\text {st }}$ Convolutional layer will be the input to the next Convolutional layer. The Convolutional functions are deployed according to Eq. (1) and Eq. (2). The output is generated from the $2^{\text {nd }}$ Convolutional layer with feature maps of the max-pooling layer. Even though the operation is performed using the feature of the location in the Convolutional layer, the computational complexity of the entire feature map will reduce the accuracy of classification. The max-pooling operation can segregate the input feature value into a group of operations. Therefore, the highest value is computed from the feature map. The $\mathrm{N} \times \mathrm{N}$ matrix is used to compute the feature map using Eq. (3). 


$$
i w_{j}=\max \left(i w_{i}^{n \times n} w f(n, n)\right)
$$

The next stages of the operations are equal to the initial stage of the Convolutional layer excluding the intensity of the feature maps. The output value of every stage has the spatial value and feature map. The feature map must consist of an initial level of features like simple operations. The prime importance of increasing the layers is to improve the visual impacts with the minimum amount of representation. The Fully Connected Networks are used to classify the features captured in the Convolutional layer. The hidden layers are used to generate the expected output of the max-pooling layer. The computation is performed using the hidden layer of Fully Connected Networks in Eq. (4) and Eq. (5).

$$
\begin{aligned}
& h_{\text {lin }}=\operatorname{fun}\left(W t_{h 1}, \operatorname{Bias}_{h 1}\right) \\
& h_{\text {lin }}=\operatorname{Bias}_{h 1}+\sum_{j=1}^{L} W t_{j} C_{j}
\end{aligned}
$$

Convolutional Networks employs by moving small filters through the input image. This means the filters are re-used for recognizing patterns throughout the whole input image. This makes the Convolutional Networks much more authoritative than Fully-Connected networks with a similar number of variables. This, in turn, makes the Convolutional Networks quicker to train. The ReLU based Activation function is generated using the threshold value of zero with Eq. (6).

$$
h_{l}=\operatorname{fun}\left(h_{\text {lin }}\right)=\max \left(0, h_{\text {lin }}\right)
$$

Likewise, the activated output values for the nodes in the adjacent layer are generated using the hidden layers. The output of the CNN layer is stopped at output layers contains the total amount of nodes that describe the assumed amount of class labels to be identified. The input pixel is categorized with a Softmax activation function. The input pixel is classified and compared with the probability output values and it is generated using Eq. (7).

$$
\operatorname{Pro}_{i}=\frac{e^{\beta i}}{\sum_{i=1}^{n} e^{\beta i}}
$$

where $\beta_{i}=\left(\beta_{1}, \beta_{2}, \ldots, \beta_{n}\right)$ is the input for the Softmax activation function for the output layer. The error within the assumed and the generated output is computed with the activation function. For the training stage, the derivation for getting the error value is computed using the weight function with the help of fully connected networks. The entropy miss function $\delta(\varnothing)$ is implemented to find the probability function using Eq. (8).

$$
\delta(\varnothing)=-\frac{1}{Z} \sum_{n=1}^{Z} \sum_{k=1}^{0} f u n\{k=P(n)\} \log T_{k}(n)
$$

The symbols used to construct the proposed methodology are illustrated in Table $\mathbf{1}$.

Table 1. Symbols used in the proposed methodology

\begin{tabular}{cc}
\hline Symbol & Description \\
\hline$h_{\text {lin }}$ & Hidden layer for linear values. \\
\hline Bias $_{h 1}$ & Bias value for the hidden layer 1 \\
\hline$C_{j}$ & Max pooling layer input value \\
\hline$W t_{j}$ & Weighted value \\
\hline Feature \\
\hline$i w_{j}$ & Feature Map \\
\hline$w f(n, n)$ & The highest value of the adjacent window of N $\mathrm{N}$ window \\
\hline$i w_{i}^{n \times n}$ & Window function of the feature map \\
\hline$A c t_{i}^{l}$ & Position of the input window for ith feature map \\
\hline$A c t_{i}^{l-1}$ & Activation for the feature map in layer \\
\hline$w t_{i, j}^{l}$ & Activation for the feature map for $l-1$ layer in the layer \\
\hline Bias $_{i}^{l}$ & Filter kernel level of Convolutional layer \\
\hline
\end{tabular}




\begin{tabular}{cc}
\hline$*$ & Operator for Convolutional layer \\
\hline$Z$ & The total amount of training patches \\
\hline$P(n)$ & Training sample \\
\hline$T_{k}(n)$ & The conditional probability of the particular sample \\
\hline$f u n\{k=P(n)\}$ & indicative function \\
\hline
\end{tabular}

\subsection{The new algorithm}

\section{Step 1: Building the framework}

i. Construct the CNN model in the process of the Convolutional layer with the hidden layers for getting better output.

ii. Apply filters to each layer with applied window size to every Maxpool layer.

iii. The ReLU activation function is the frame for each component of the Convolutional layer and hidden layer. Frame Softmax activation function for the output layer in Fully Connected Networks.

\section{Step 2: Dataset preparation}

i. The pixel values of the input image are normalized to produce the scaling with the value of (0.0 to 255.0) to (0.0 to 1.0).

ii. According to the band computation, the patch image is computed for every pixel value. Read the truth value for every input pixel and organize them into the target class. Repeat the same procedure for every pixel in the input classification.

iii. Perform the shuffling operations within the pixels for each target class.

iv. Divide the total number of pixels into two parts where $75 \%$ is used for training and $25 \%$ is used for testing using Convolutional Neural Networks.

\section{Step 3: CNN model initialization}

i. Every filter kernel is used the fully connected networks with bias values and weight values for random units.

ii. The maximum number of epochs is utilized for training the batches with striding and zero padding concepts.

iii. The value of loss function is computed for further processing.

\section{Step 4: Training Process}

i. For every epoch, the input value for every patch in the network is used to calculate the output layer values.

ii. For every output layer, calculate the conditional probability and loss function.

iii. Update the value of the weight of every network with the learning ratio and the kernel value for the input.

iv. Compute the backpropagation value for the Convolutional layer and hidden layers and the bias values for fully connected networks.

\section{Step 5: Testing Process}

i. The patches for every testing sample are integrated into the Convolutional neural network to eliminate the weight of the patches.

ii. The errors for generating the classification are computed in every process.

iii. The process is terminated whenever the accuracy value is reached the threshold value.

\section{Step 6: Implementing the Final output model}

i. While producing the output value, the accuracy is the prime parameter. The usual parameters like learning ratio, cost function, number of filters in the Convolutional layers, Maxpooling value, total amount of hidden layers, size of the patches, combined layers formation. 
ii. The produced output value while classification is in the Testing and Training process. The optimized weight values can be used for generating the best model.

\subsubsection{Pre-processing}

The preprocessing pipeline is the following:

1. Download all images.

2. Crop \& resize all images

3. Rotate \& mirror all images.

4. Convert all images to an array of NumPy arrays.

\subsubsection{Download All Images}

The images were downloaded using the Kaggle CLI. Running this on an EC2 instance allows downloading the images in about 30 minutes. All images are then placed in their respective folders and expanded from their compressed files. In total, the original dataset totals 35 gigabytes.

\subsubsection{Crop and Resize All Images}

All images were scaled down to 256. Despite taking longer to train, the detail present in photos of this size is much greater than 128. Additionally, 403 images were dropped from the training set. Scikit-Image raised multiple warnings during resizing due to these images having no color space. Because of this, any images that were completely black were removed from the training data.

\subsubsection{Rotate and Mirror All Images}

All images were rotated and mirrored. Images exclusive of retinopathy were mirrored. Images that obligated retinopathy was mirrored and rotated with the following degrees such as 90, 120, 180, and 270. The first images illustrate two pairs of eyes, along with the black borders. Figure 3 demonstrates all kinds of Retinopathy images.
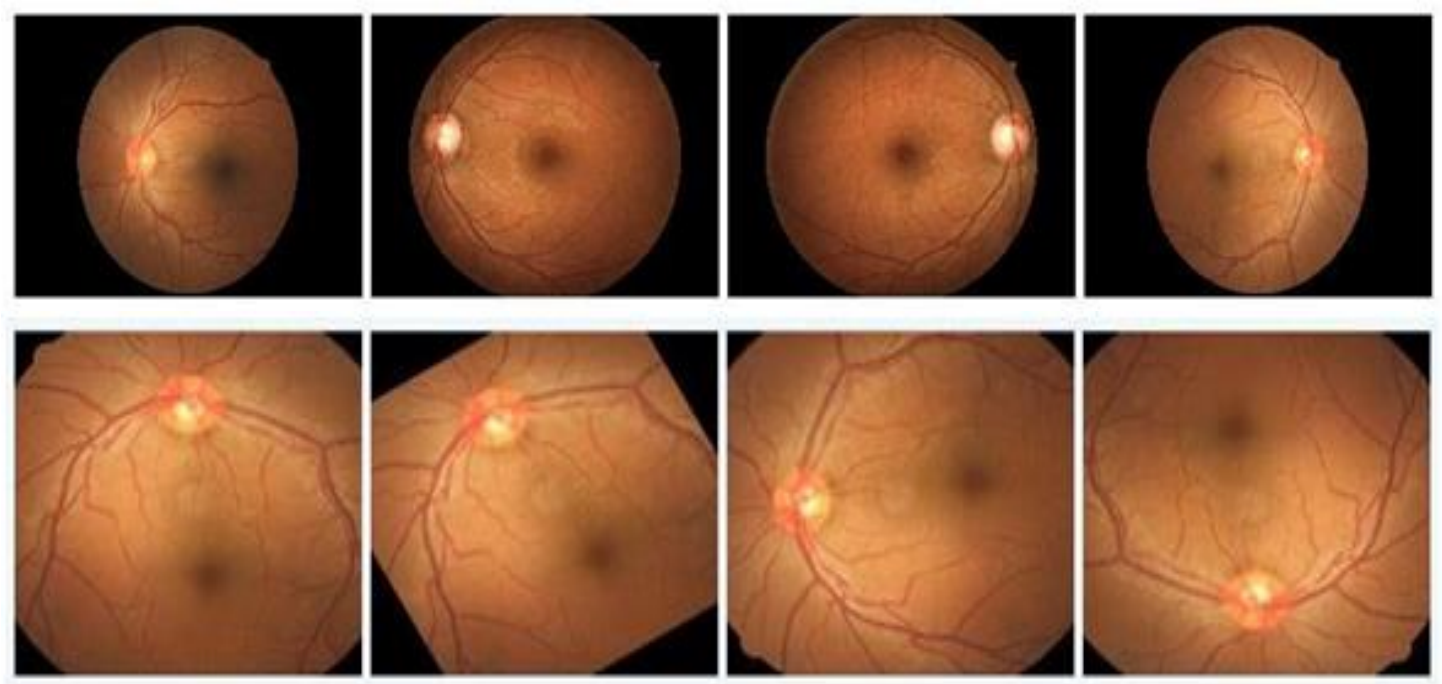

Figure 3. Eye Retinopathy images.

After rotations and mirroring, the class difference is rectified; with a little thousand more images developing retinopathy. Overall, there are 106,386 images being processed by the neural network.

The framework for the proposed methodology for image classification is demonstrated in Figure 4. The proposed methodology uses the Convolutional Neural Network with the architecture of the layers for training and validating the accuracy level. The sharing of weight coefficient in 
Convolutional layers may minimize the number of parameters. It is much easy to identify the edges and other corners. The pooling layer is used to identify the location of the extracted images.

Input Image

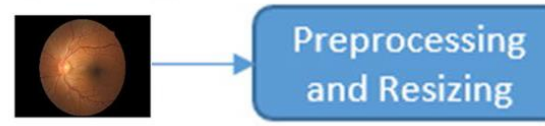

Input Layer

Convolutional Layer

ReLU Layer

Max-Pooling Layer

Convolutional Layer

ReLU Layer

Max-Pooling Layer

Convolutional Layer

ReLU Layer

Convolutional Layer

ReLU Layer

Max-Pooling Layer

Fully-Connected Layer

ReLU Layer

Fully-Connected Layer

ReLU Layer

Dropout Layer

Softmax Layer

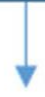

Classification

Figure 4. Framework for the proposed methodology.

\section{Experiments}

\subsection{Datasets}

Kaggle Retinopathy detection dataset [39] is used to analysis the Retina images to compare with the existing methodologies. After pre-processing is done, the subsets of the dataset are divided into a training set and a testing set. The training set is with 45000 images, and the testing set contains nearly 8500 images. It is illustrated in Figure 5. 


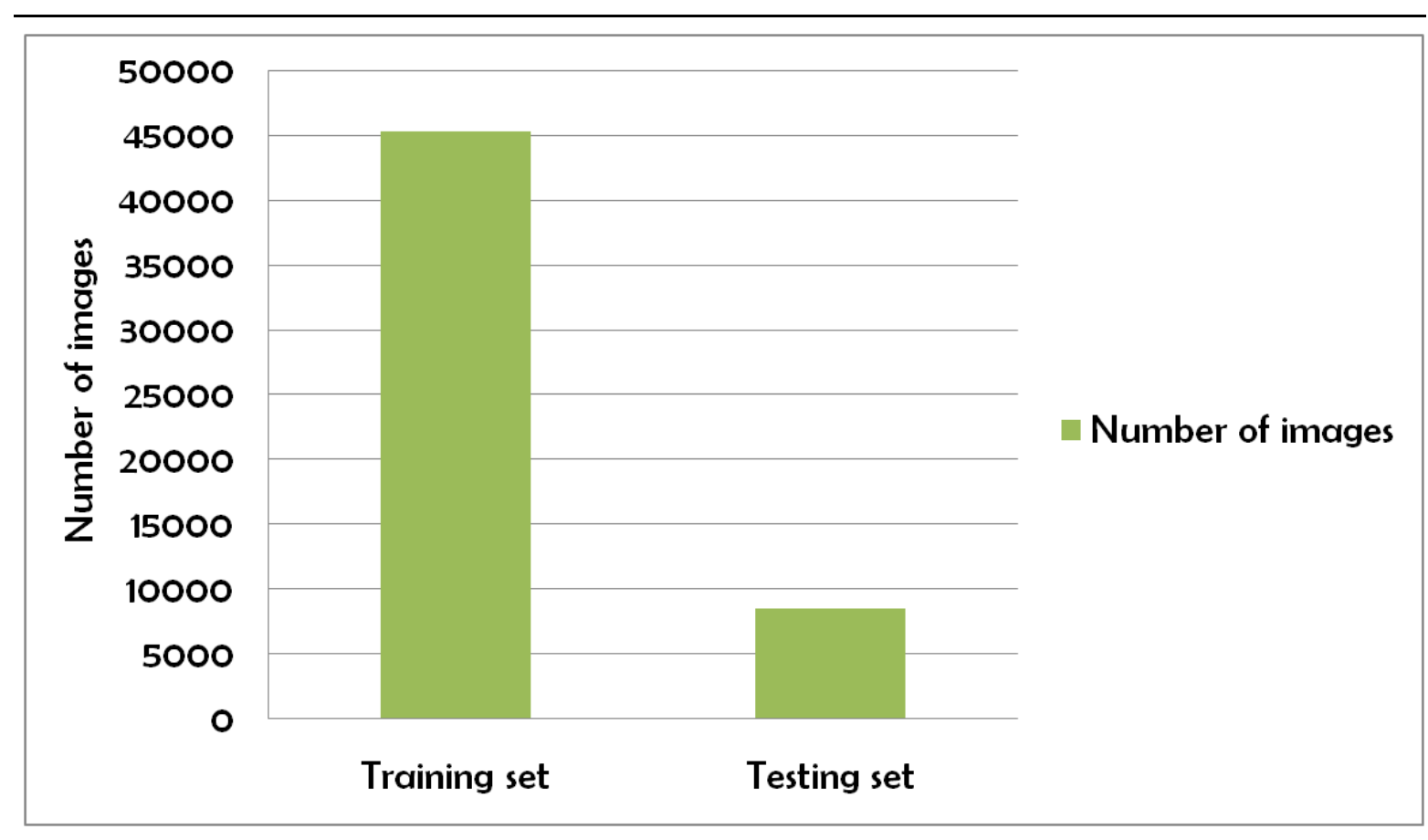

Figure 5. Subset of Kaggle Retinopathy detection dataset.

\subsection{Configuration}

The proposed methodology is compared with the related methods namely KNN [35], GD-CNN [17], SVM [37] and DREAM [40]. Table 2 demonstrates the proposed methodology with Convolutional neural network layers and the related configurations.

Table 2. Configuration for layers.

\begin{tabular}{cc}
\hline Layers & Configuration \\
\hline Input image & $227 \times 227 \times 3$ \\
\hline Convolution & $48 \times 48 \times 96$ \\
\hline size of the kernel & $11 \times 11$ \\
\hline kernel stride & 4 \\
\hline MaxPooling & $3 \times 3$ \\
\hline Fully Connected networks & 4096 \\
\hline SoftMax & 6 \\
\hline
\end{tabular}

\subsection{Precision}

Precision is a measure that tells us what proportion of patients that we diagnosed are having retinopathy, actually had retinopathy. The predicted positives and the people actually having retinopathy are TP. The computation of Precision is demonstrated in Eq. (9).

$$
\text { Precision }=\frac{T P}{T P+F P}
$$

\subsection{Recall}

The recall is a measure that tells us what proportion of patients that actually had retinopathy was diagnosed by the algorithm as having retinopathy. The actual positives and the people diagnosed by the model having retinopathy are TP. The Recall is computed using Eq. (10).

$$
\text { Recall }=\frac{T P}{T P}+F N
$$

\section{5. $F 1_{\text {Score }}$}


It is computed with the weighted average values of precision and recall parameters. Additionally, these parameters combine false positive and negative values. It is also used to identify the accuracy level within every class distribution. Accuracy is computed with similar values of false positives and negatives. It is computed using Eq. (11).

$$
F 1_{\text {score }}=2 * \frac{(\text { Recall } * \text { Precision })}{(\text { Recall }+ \text { Precision })}
$$

\subsection{Cohen's kappa coefficient $(\kappa)$}

This parameter will process the category-based values to implement the agreement computation within the metrics. It is also computed with the correlation value based on the patient characterization using different kinds of machine learning methods which will identify the risk parameters of DR.

\subsection{Results}

The simulation is conducted using Anaconda and comparative performance analysis is conducted. Prediction for the image is computed by showing the retinography starting stage with exploratory data analysis from the following Figures $\mathbf{6}$ to $\mathbf{1 0}$

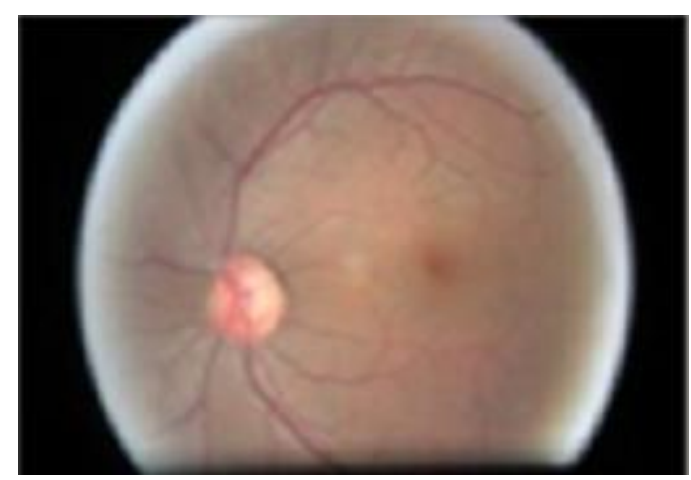

Figure 6. Prediction for the image which is unclear in Right side.

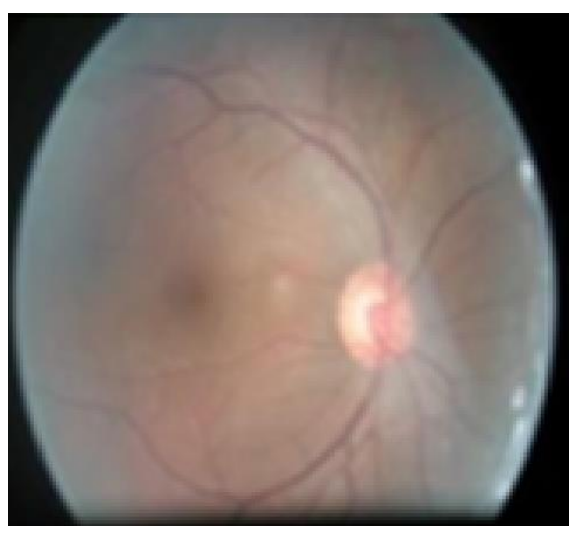

Figure 7. Prediction for the image which is unclear in the left side. 


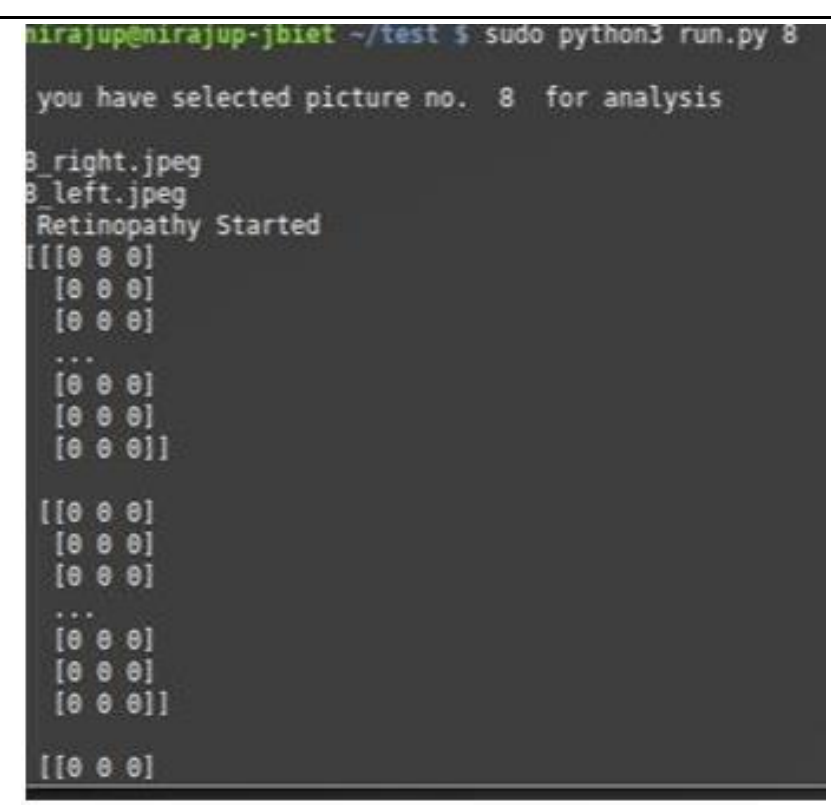

Figure 8. Prediction for the image for Retinopathy starting stage.

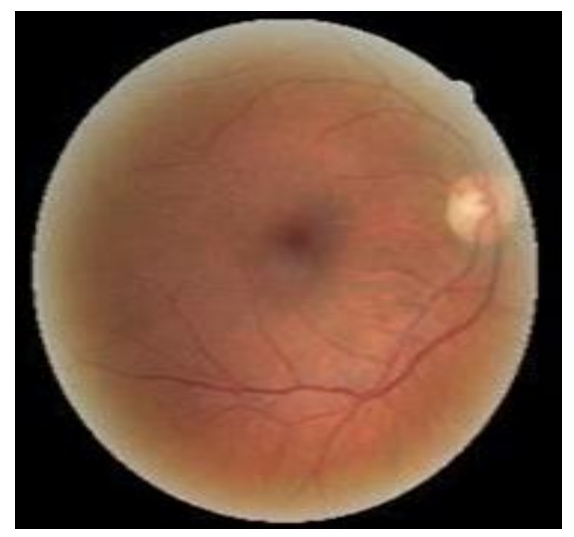

Figure 9. Prediction for Retinopathy starting stage of Right side.

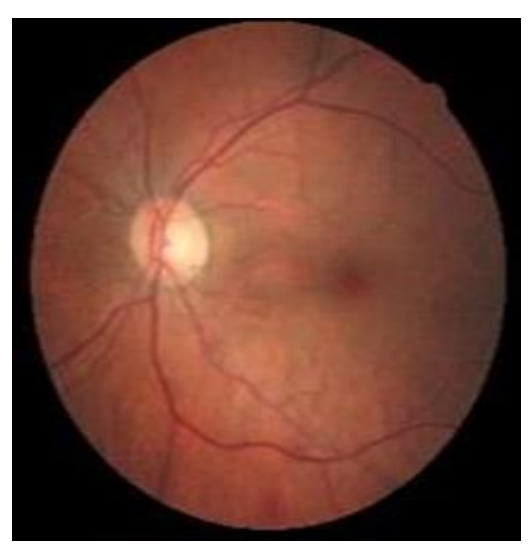

Figure 10. Prediction for Retinopathy starting stage of Left side. 


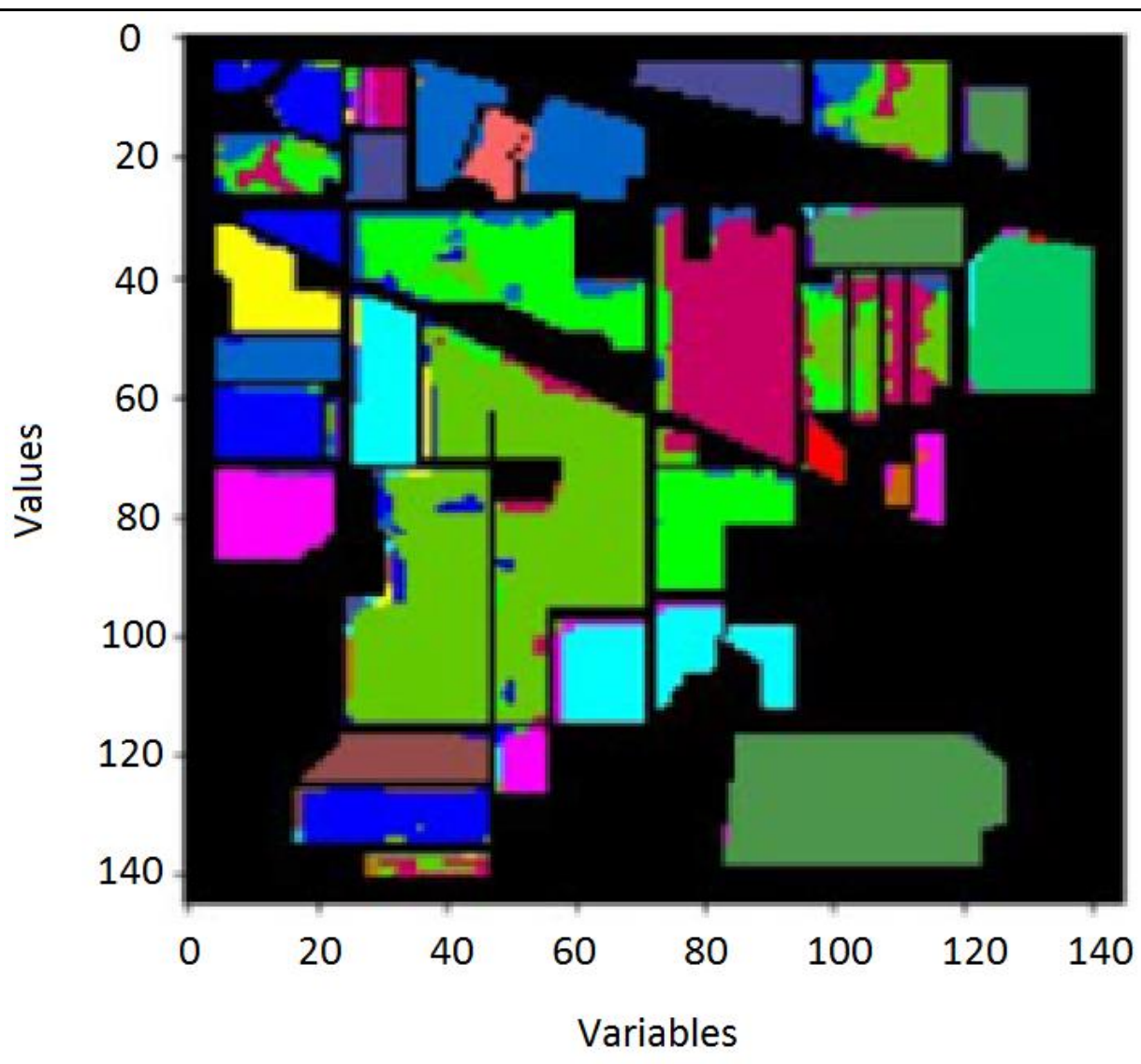

Figure 11. Accuracy with variables and values for input image.

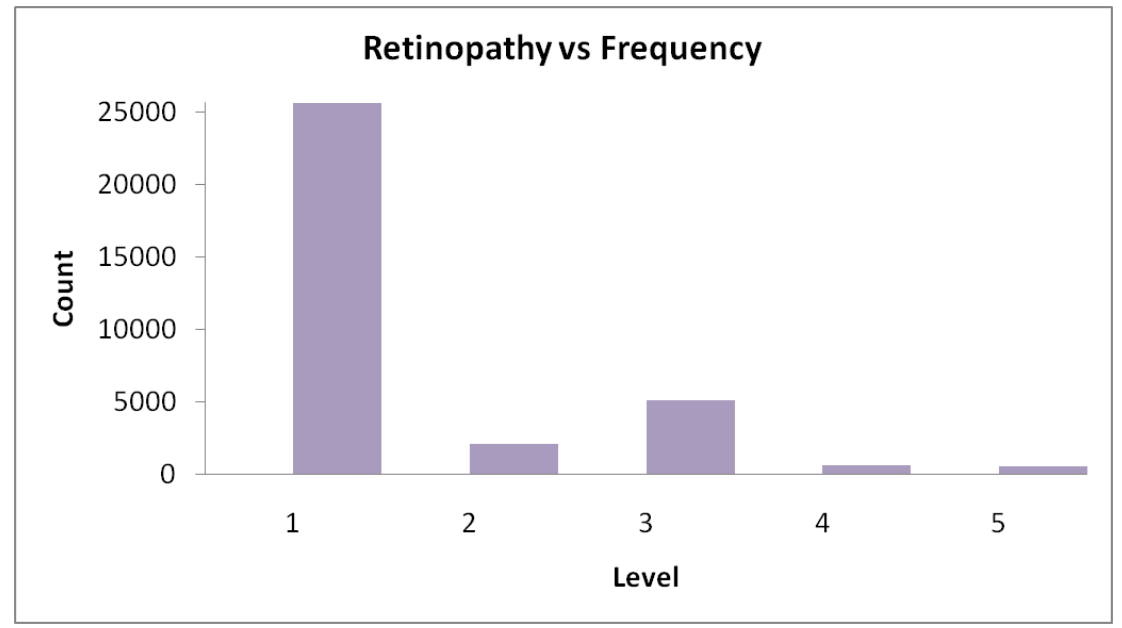

Figure 12. Exploratory Data Analysis (EDA).

The above EDA gives the full structure of DR identification when the new input inserted; it changes the Level labels for binary classification. Thus, either the person has a degree of retinopathy or not. Figure $\mathbf{1 1}$ demonstrates the Accuracy with variables and values for the input image and Figure 12 illustrates Exploratory Data Analysis (EDA). 


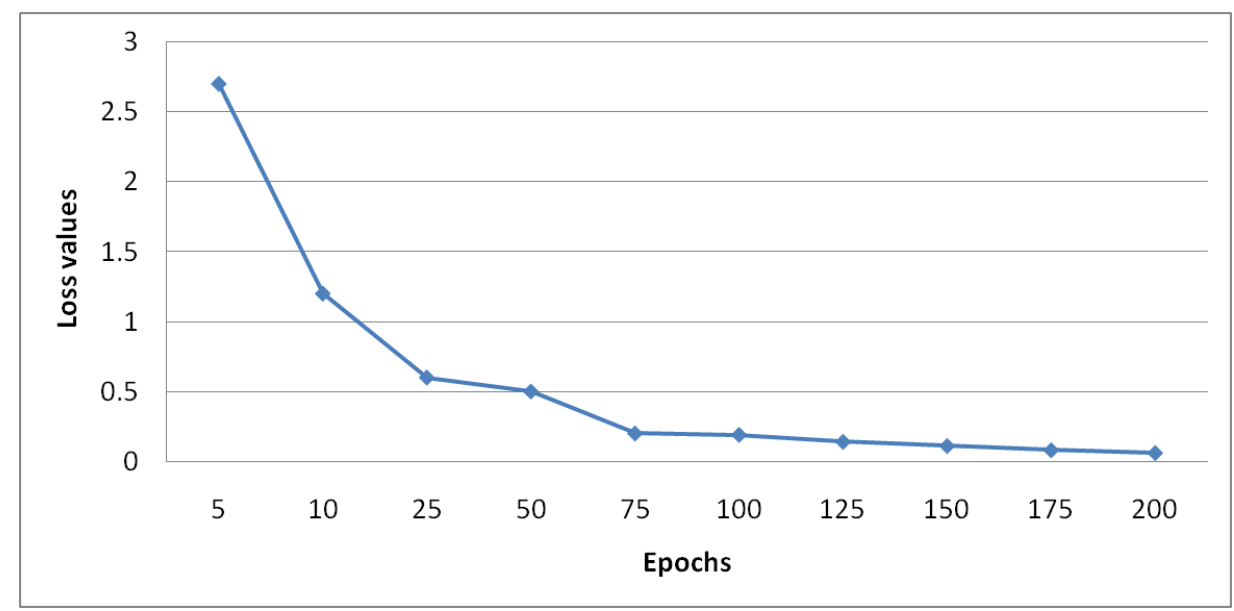

Figure 13. Loss of training dataset.

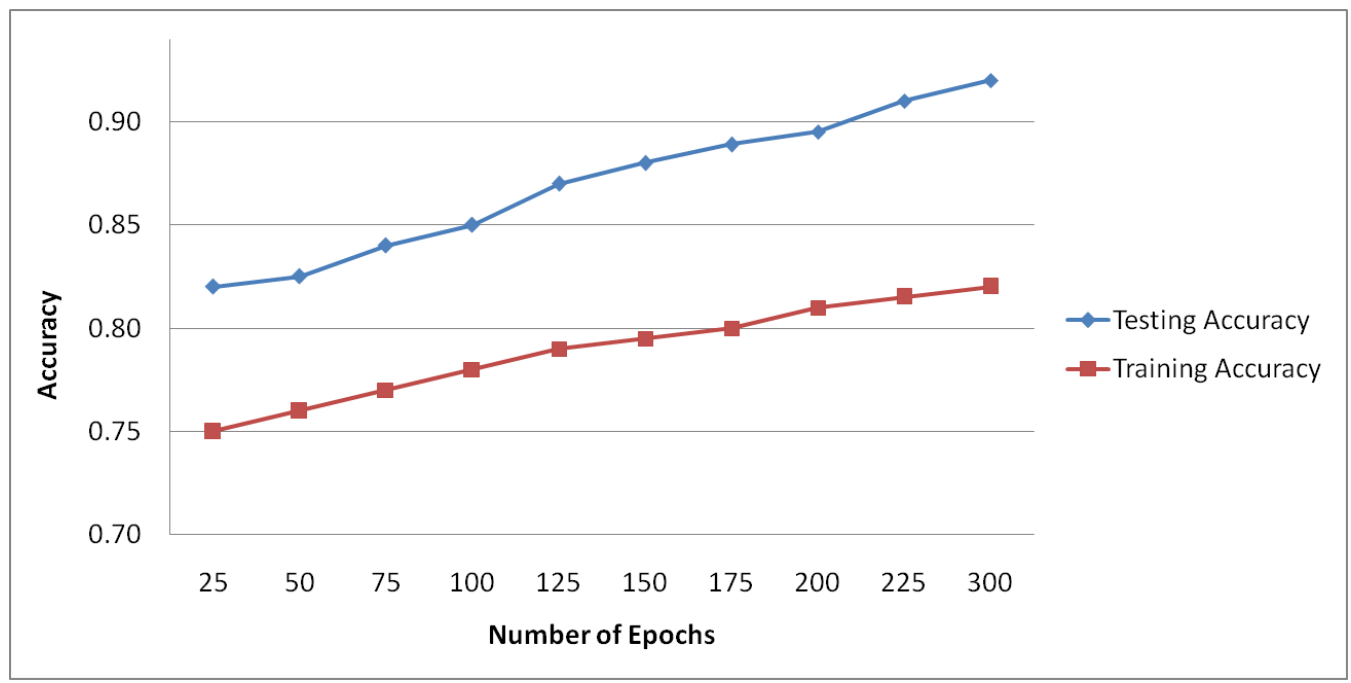

Figure 14. Overall accuracy.

The classification performance is computed using the overall accuracy. There were several classes that have some known samples, like similarity images. To increase the number of training samples, the classes with the minimum number of samples are used for classification. The results generated from the experiments in several numbers of epochs. It is concluded that the more amount epochs are needed to improve the accuracy level. The accuracy for testing is minimum value concerning the training accuracy, the visual representation of the input image and the assumed image has been used with the period of 25 epochs. Figure 13 demonstrates the Loss of training dataset, and Figure 14 illustrates the overall accuracy. Figure 15 states that the Loss value for the dataset for retinopathy images. 


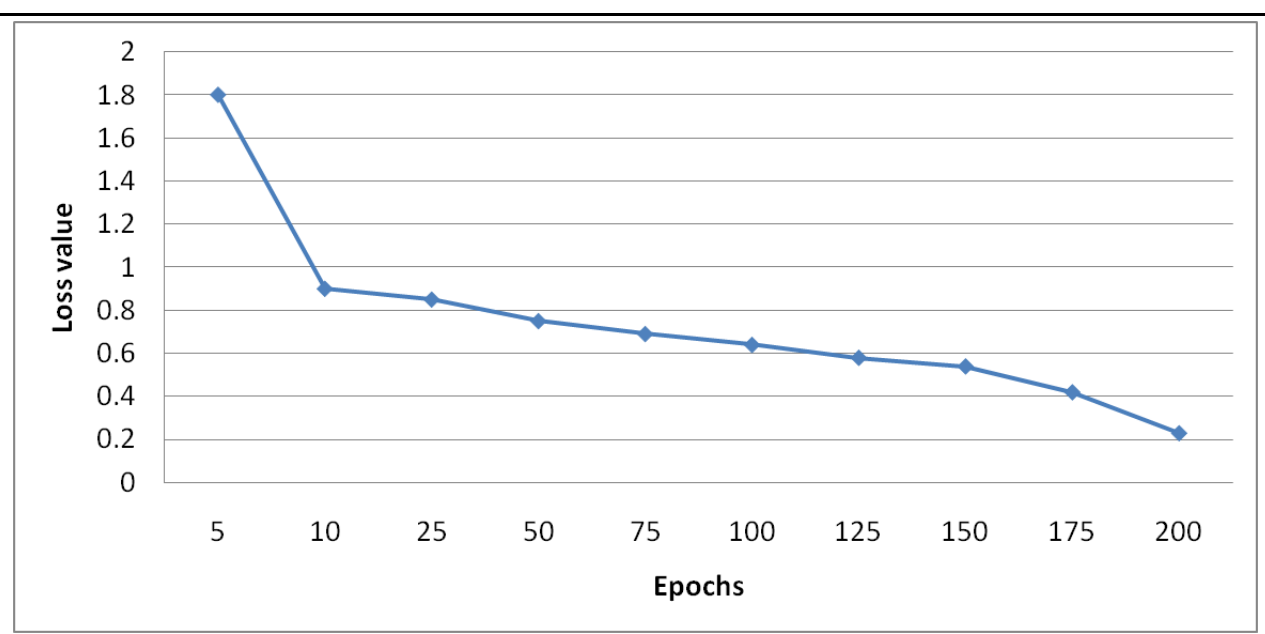

Figure 15. Loss value.

The predicted image that has the minimum amount of classification is implemented. The proposed algorithm has a better classification for high frequency-based input images. The overall accuracy is maintained through the proposed methodology for training is $98.45 \%$ and testing is $92.15 \%$.

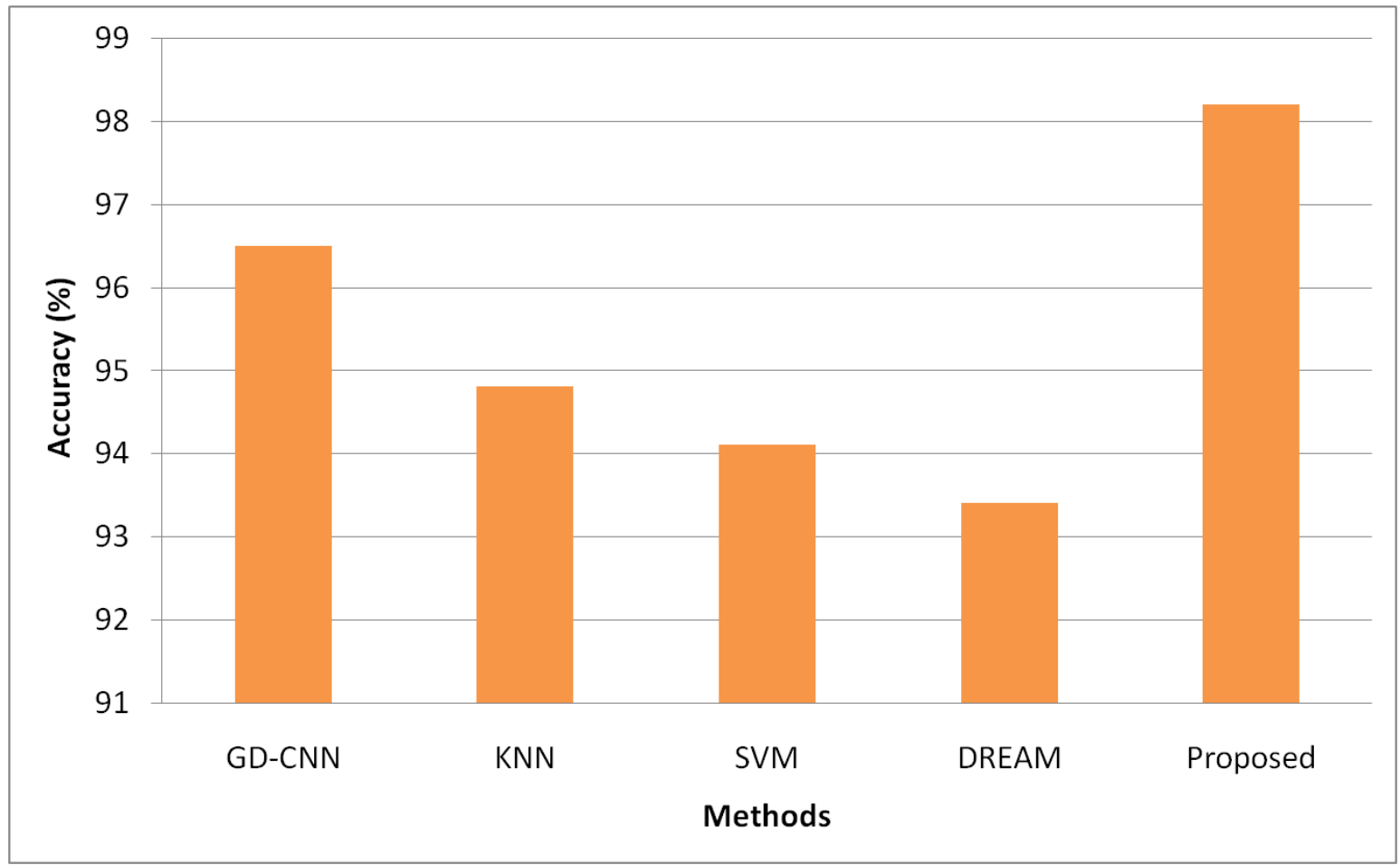

Figure 16. Accuracy (\%). 


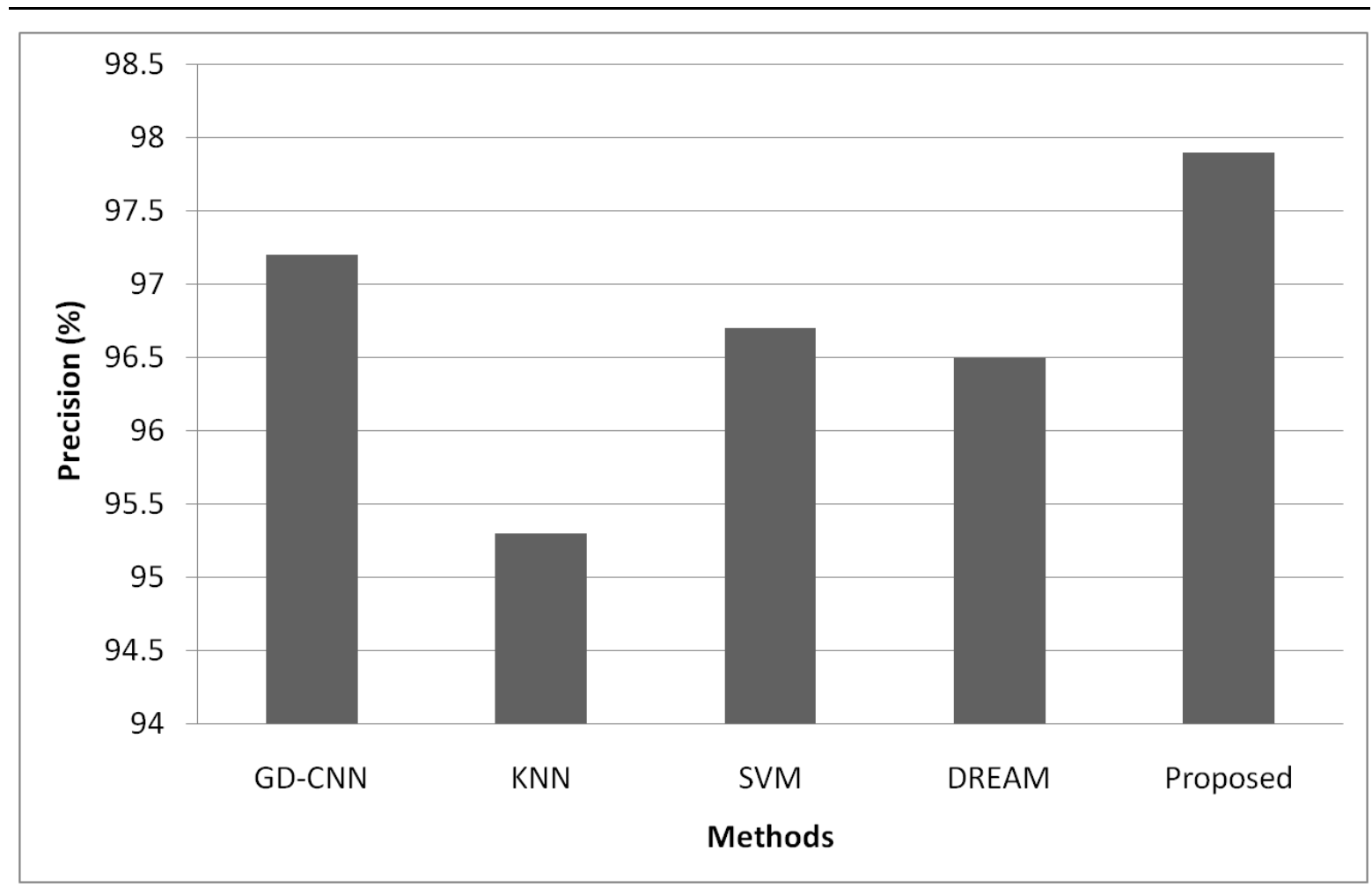

Figure 17. Precision (\%).

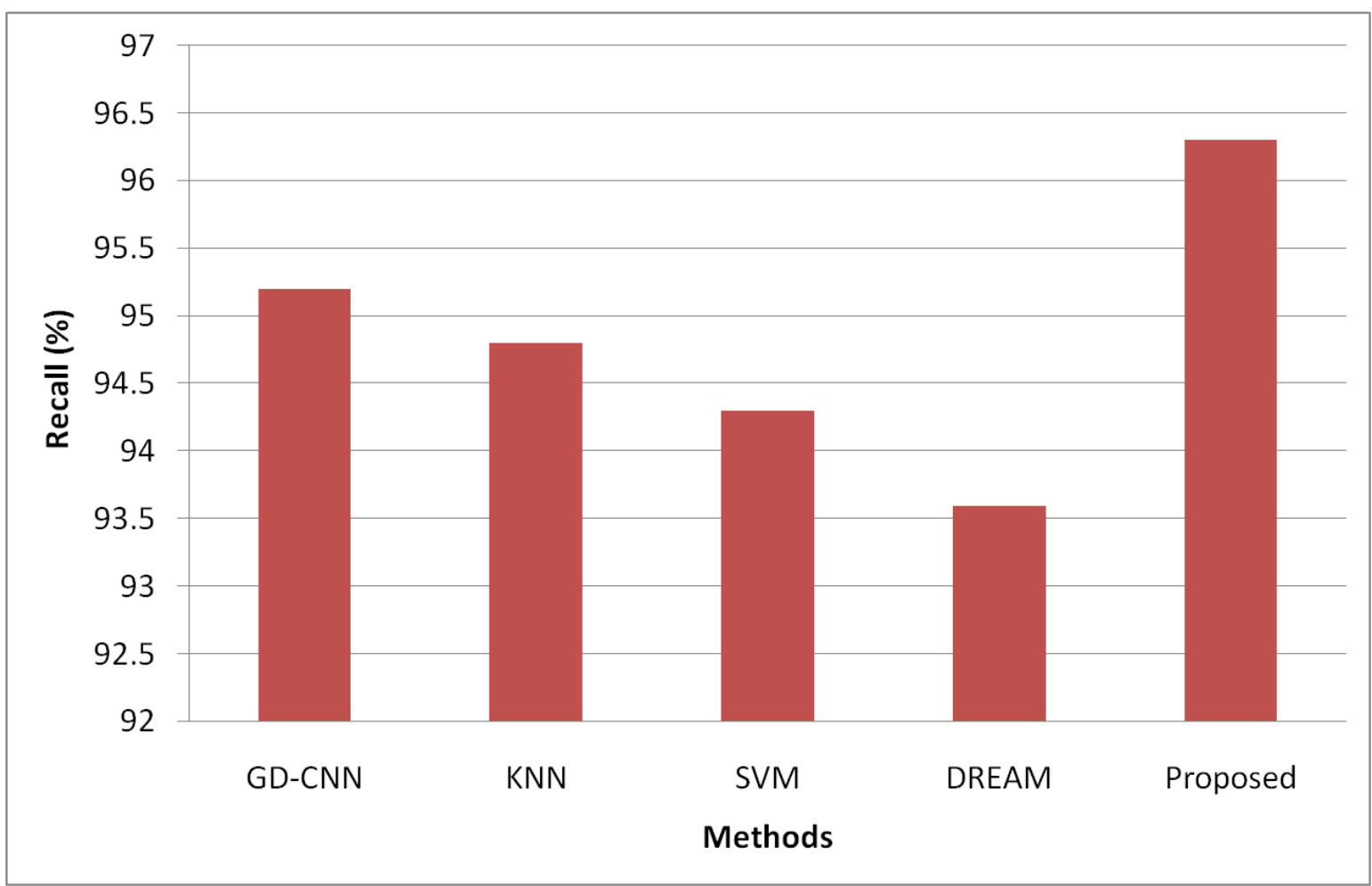

Figure 18. Recall (\%).

The proposed methodology is compared with the related methods of DREAM, KNN, GD-CNN and SVM. The results proved that the proposed methodology has improved performance compared to the performance metrics of Accuracy, Precision, Recall and $F 1_{\text {score }}$. Figure 16 demonstrates the 
Accuracy \%, Figure 17 demonstrates the Precision \%, Figure 18 demonstrates the Recall \%, and Figure 19 demonstrates $F 1_{\text {score }} \%$.

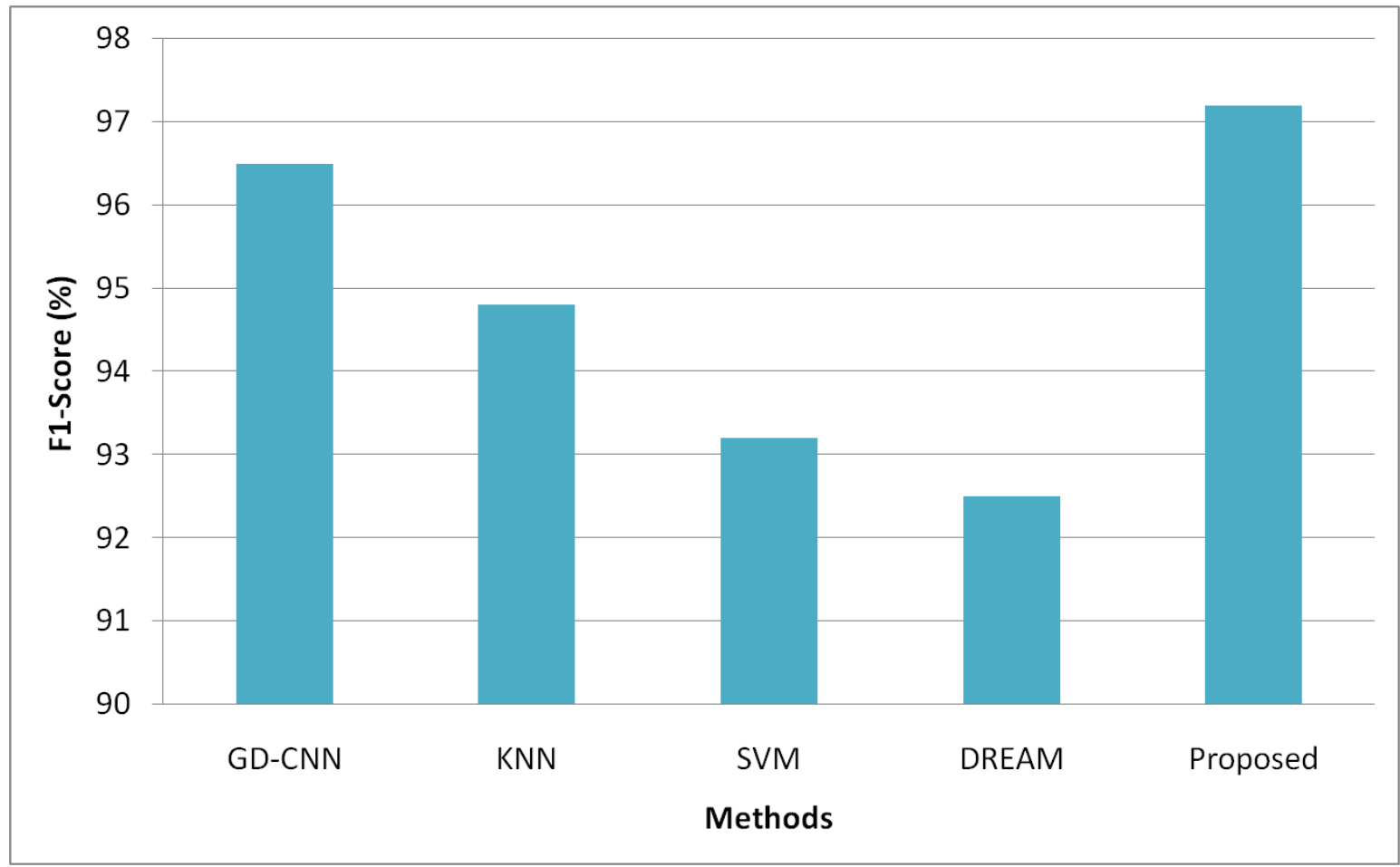

Figure 19. $F 1_{\text {score }}(\%)$

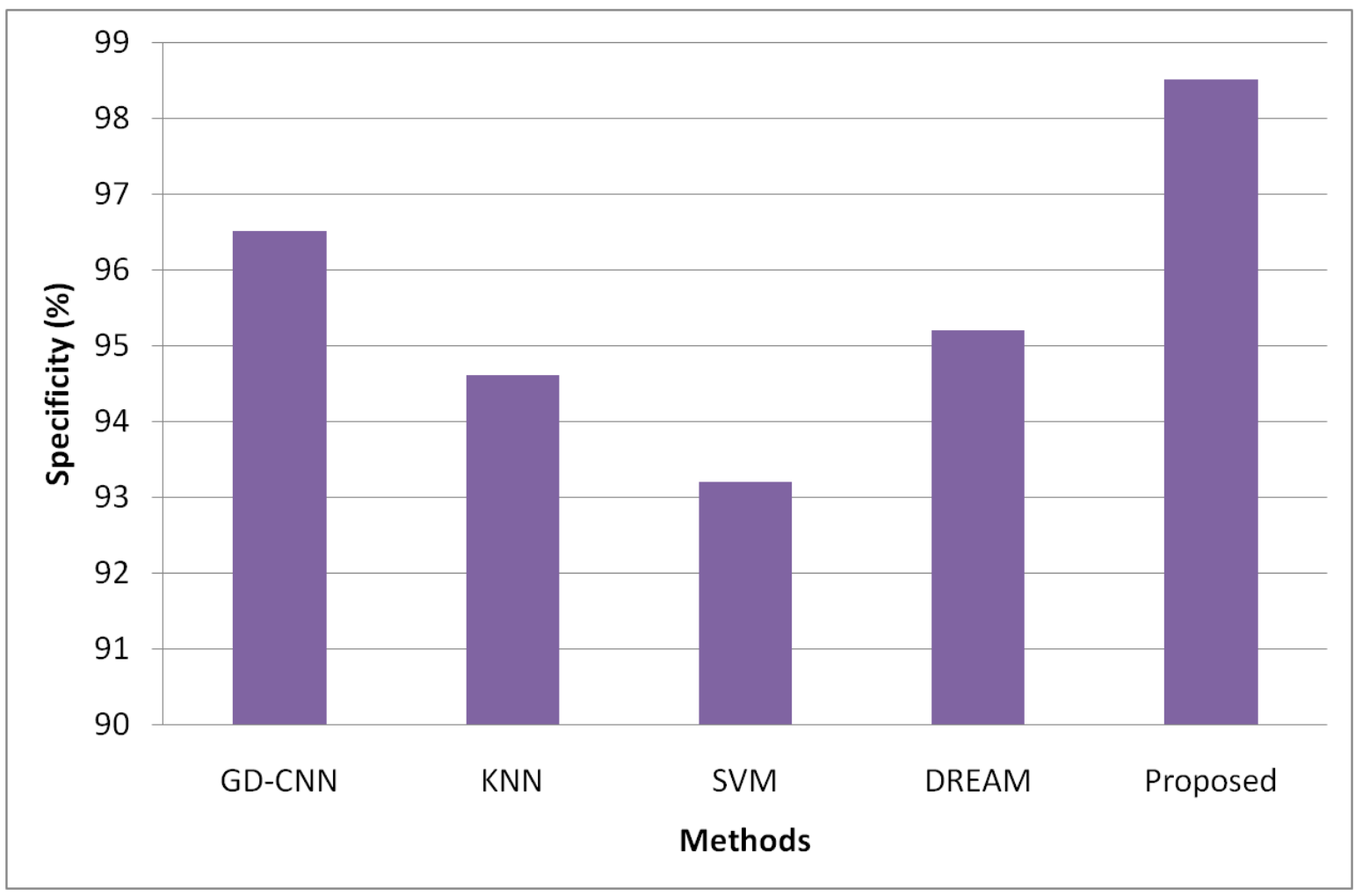

Figure 20. Specificity \%

Specificity could be measured using the proportion of exact negatives that are identified as the condition value to diagnosis the diabetic retinopathy from the images and the simulation results are 
presented in Figure 20. It suggests that the proposed methodology has the improved Specificity compared with the related methods. The proposed Convolutional Neural Networks are constructed to compute the semantic data within the adjacent pixels. The proposed method is having the enhanced amount of quicker processing. It can process the large amount of training data for identifying the diabetic retinopathy. The hyper parameter tuning is successfully performed in every input image. The activation maps are organized with filter value. The rectangle is framed for every single patches, the input image is used to filter passes. A fully connected layer is developed to produce the output with every single node. The proposed method can extract the features automatically from the images and also the pre-trained function is not compulsory for classification.

The precision value is measured that the proportion of the diagnosed Retinopathy from the input images. The results proved that the proposed method has an improved amount of percentage compared with the other models. The Recall is computed using the exact positive values for the actual diabetic Retinopathy. $F 1_{\text {score }}$ is computed based on the weighted average of precision and recall metrics and the false positives and negative values are identified. The accuracy is computed based on the parameters of precision, recall and $F 1_{\text {score }}$. The performance results showed that the proposed methodology is performed better. The Exploratory Data Analysis is computed based on the binary classification. The total amount of count is improved in every level. The loss of training dataset is reduced whenever the Epochs are increased, the overall accuracy of the Testing and Training are also measured in the total amount of Epochs.

Another metric used to compare the methods is the iteration count in the training whenever the model has achieved a full accuracy level. The number of batches for validation and testing accuracy for the proposed methodology is $94.33 \%$ and $88.32 \%$, respectively. The final metric used to compare the methods are the inclusion of the dropout layer within the Softmax classifier layer for validating the accuracy. The proposed methodology has achieved better performance compared to other methodologies.

\section{Concluding remarks}

Practically, a medical expert can recognize diabetic retinopathy by the occurrence of lesions correlated with the vascular abnormalities instigated. This methodology is efficient, but its resource anxiety is tremendous. In this work, we propose a new model that uses the Convolutional Neural Network (CNN) to increase the accuracy of Retinopathy Diagnosis. It is utilized to analyze the pathogenesis to detect diabetic retinopathy. Experiments suggested better performance of the new model.

The research work will be continually progressing. Instigating novel techniques, we expect to raise the accuracy of the technique as well as to enlarge it to different types of retinopathy and other eye diseases. We will be trying to use this model for more massive data sets. Other deep learning techniques will also be used in this project. Enlargement of a neural model centered on detection principles and capability of the meticulous discovery of restrained lesions, just like specialists do, will be achieved.

\section{Author Contributions:}

G. Arun Sampaul Thomas : Writing - original draft, Writing - review \& editing, Y.Harold Robinson : Writing original draft, Conceptualization, Data curation, Validation. E. Golden Julie: Writing - original draft, Conceptualization, Data curation, Validation.Vimal shanmuganathan: Conceptualization, Formal analysis, Writing - review \& editing, Supervision. Yunyoung Nam: Writing - review \& editing. Seungmin Rho: Formal analysis, Supervision. Writing - review \& editing

\section{Acknowledgement}

This work was supported by the National Research Foundation of Korea(NRF) grant funded by the Korea government(MSIT) (NRF-2019R1F1A1060668) and also supported by the Soonchunhyang University Research Fund. 


\section{References}

1. White NH, Sun W, Cleary P a, Tamborlane W V, Danis RP, Hainsworth DP, et al. Effect of Prior Intensive Therapy in Type 1 Diabetes on 10-Year Progression of Retinopathy in the DCCT/ EDIC : Comparison of Adults and Adolescents. Diabetes 2010;59:1244-53. doi:10.2337/db09-1216.

2. Klein R, Klein BEK. Vision disorders in diabetes. Diabetes Am 1995;2nd ed.:293-338. doi:10.1063/1.1522643.

3. X Zhang, Z Li, C C Loy. PolyNet: a pursuit of structural diversity in very deep networks. Proceedings of the IEEE Conference on Computer Vision and Pattern Recognition. 2017:3900-8.

4. M R K Mookiah, U R Acharya, H Fujita et al. Local Configuration Pattern Features for Age-Related Macular Degeneration Characterization and Classification. Comput Biol Med 2015;63:208-18.

5. The International Agency for the Prevention of Blindness. Status and Challenges in DR https://www.iapb.org/wp-content/uploads/13-Status-and-Challenges-in-DR-China -Chaoju-Group.pdf.

6. P Burlina, K D Pacheco, N Joshi, et al. Comparing humans and deep learning performance for grading AMD: A study in using universal deep features and transfer learning for automated AMD analysis. Comput Biol Med 2017;82(3):80-6.

7. U Raghavendra, H Fujita, S V Bhandary, et al. Deep convolution neural network for accurate diagnosis of glaucoma using digital fundus images. Information Sciences 2018;441:41-9.

8. V Gulshan, L Peng, M Coram, et al. Development and validation of a deep learning algorithm for detection of diabetic retinopathy in retinal fundus photographs. JAMA 2016;316(22):2402-10.

9. U T V Nguyen, A Bhuiyan, L A F Park, et al. Automatic detection of retinal vascular landmark features for colour fundus image matching and patient longitudinal study. Proceedings of 20th IEEE International Conference on Image Processing. 2013:616-20.

10. Z Zhou, J Shin, L Zhang, et al. Fine-tuning convolutional neural networks for biomedical image analysis: actively and incrementally. Proceedings of IEEE Conference on Computer Vision and Pattern Recognition. 2017:4761-72.

11. Z Zhang, Y Xie, F Xing, et al. MDNet: A semantically and visually interpretable medical image diagnosis network. Proceedings of IEEE Conference on Computer Vision and Pattern Recognition. 2016: 3549-57.

12. D Xiao, S Yu, J Vignarajan, et al. Retinal hemorrhage detection by rule-based and machine learning approach. Proceedings of 39th Annual International Conference of the IEEE Engineering in Medicine and Biology Society. 2017:660-3.

13. J I Orlando, E Prokofyeva, M D Fresno, et al. An ensemble deep learning based approach for red lesion detection in fundus images. Comput Methods Programs Biomed 2018;153:115-27.

14. S Roychowdhury, D D Koozekanani, K K Parhi. DREAM: diabetic retinopathy analysis using machine learning. IEEE J Biomed Health Inf 2014;18(5):1717-28.

15. D S W Ting, C Y Cheung, G Lim, et al. Development and validation of a deep learning system for diabetic retinopathy and related eye diseases using retinal images from multiethnic populations with diabetes. JAMA 2017;318(22):2211-23.

16. A Krizhevsky, I Sutskever, G E Hinton. ImageNet classification with deep convolutional neural networks. Proceedings of 26th Annual Conference on Neural Information Processing Systems. 2012: 1097- 105.

17. G Huang, Z Liu, L Maaten, et al. Densely connected convolutional networks. Proceedings of the IEEE Conference on Computer Vision and Pattern Recognition. 2017:2261-9.

18. Joly, A., Goëau, H., Glotin, H., Spampinato, C., Bonnet, P., Vellinga, W.-P., et al. (2015). LifeCLEF 2015: multimedia life species identification challenges. In J. Mothe, J. Savoy, J. Kamps, K. Pinel-Sauvagnat, G. Jones, E. San Juan, L. Capellato, \& N. Ferro (Eds.), Experimental IR meets multi-linguality, multimodality, and interaction. Lecture notes in computer science. Cham: Springer.

19. Khalifa, N. E., Taha, M. H., Hassanien, A. E., \& Selim, I. (2018). Deep Galaxy V2: Robust deep Convolutional neural networks for galaxy morphology classifications. In 2018 IEEE international conference on computing sciences and engineering, ICCSE, IEEE (pp. 122-127).

20. S Xie, R Girshick, P Dollár. Aggregated residual transformations for deep neural networks. Proceedings of the IEEE Conference on Computer Vision and Pattern Recognition. 2017:5987-95. 
21. J Hu, L Shen, G Sun. Squeeze-and-excitation networks. Proceedings of the IEEE Conference on Computer Vision and Pattern Recognition. 2018:7132-41.

22. G.Lim, M.L.Lee, W.hsu, “Transformed Representations for Convolutional Neural Networks in Diabetic Retinopathy Screening", Modern Artificial Intelligence for Health Analytic Papers from the AAAI (2014).

23. M R K Mookiah, U R Acharya, H Fujita et al. Local Configuration Pattern Features for Age-Related Macular Degeneration Characterization and Classification. Comput Biol Med 2015;63:208-18.

24. Yi-Peng Liu, Zhanqing Li, Cong Xu, Jing Li, (2019), Ronghua LiangReferable Diabetic Retinopathy Identification from Eye Fundus Images with Weighted Path for Convolutional Neural Network, Artificial Intelligence in Medicine, Elsevier.

25. M Lin, Q Chen, S Yan. Network in network. CoRR abs/1312.4400. http://arxiv.org/abs/1312.4400.

26. Pedro Costa, Adrian Galdran et al.,"A weakly supervised framework for interpretable Diabetic Retinopathy detection on Retinal images", IEEE - Advanced signal processing methods in Medical imaging, March 2018, Pg.18747-18758.

27. Kedir M.Adal, Peter G.Ven Elten et al., "An automated system for the detection and classification of Retinal changes due to Red Lesions in Fundus images", IEEE Transactions on Biomedical Engineering, September 2017, Pg. 1382-1390.

28. S. Wang, et al, "Hierarchical retinal blood vessel segmentation based on feature and ensemble learning", Neurocomputing (2014).

29. Mrinal Haloi, "Improved Microaneurysm detection using Deep Neural Networks", Cornel University Library (2015), arXiv:1505.04424.

30. M. Melinscak, P.Prentasic, S.Loncaric, "Retinal Vessel Segmentation using Deep Neural Networks", VISAPP(1), (2015):577-582

31. S. Roychowdhury, D.D. Koozekanani, Keshab K. Parhi, “DREAM: Diabetic Retinopathy Analysis Using Machine Learning", IEEE Journal of BioMedical and Health Informatics, Vol.18, No 5, September (2014).

32. J. Lachure, A.V. Deorankar, S. Lachure, S. Gupta, R. Jadhav, “Diabetic Retinopathy using Morphological operations and Machine Learning", IEEE International Advance Computing Conference (IACC), (2015).

33. R.Priya, P.Aruna, "SVM and Neural Network based Diagnosis of Diabetic Retinpathy", International Journal of computer Applications (00975-8887), volume 41- No.1,(March 2012).

34. S.Giraddi, J Pujari, S.Seeri, "Identifying Abnormalities in the Retinal Images using SVM Classifiers", International Journal of Computer Applications (0975-8887), Volume 111 - No.6,(2015).

35. Daniel Carlos Guimarães Pedronette, Ying Weng, Alexandro Baldassin, Chaohuan Hou, Semi-supervised and active learning through Manifold Reciprocal kNN Graph for image retrieval, Neurocomputing, Volume 340, 7 May 2019, Pages 19-31.

36. Hailiang Li, Kin-Man Lam, Miaohui Wang, Image super-resolution via feature-augmented random forest, Signal Processing: Image Communication, Volume 72, 2019, Pages 25-34.

37. Jingchao Cao, Shiqi Wang, Ran Wang, Xinfeng Zhang, Sam Kwong, Content-oriented image quality assessment with multi-label SVM classifier, Signal Processing: Image Communication, Volume 78, October 2019, Pages 388-397.

38. Liangxiao Jiang, Lungan Zhang, Liangjun Yu, Dianhong Wang, Class-specific attribute weighted naive Bayes, Pattern Recognition, Volume 88, April 2019, Pages 321-330.

39. https://www.kaggle.com/c/diabetic-retinopathy-detection/data

40. Roychowdhury S, Koozekanani DD, Parhi KK. DREAM: diabetic retinopathy analysis using machine learning. IEEE J Biomed Heal Informatics. 2014; 18:1717-1728.

41. Gulshan, V., Peng, L., Coram, M., Stumpe, M.C., Wu, D., Narayanaswamy, A., Venugopalan, S., Widner, K., Madams, T., Cuadros, J. and Kim, R., 2016. Development and validation of a deep learning algorithm for detection of diabetic retinopathy in retinal fundus photographs. Jama, 316(22), pp.2402-2410.

42. Gargeya, R. and Leng, T., 2017. Automated identification of diabetic retinopathy using deep learning. Ophthalmology, 124(7), pp.962-969." Please also be aware that individual eye anatomy related to myopia can affect eye disease diagnostic accuracy.

43. Lim, L.S., Lamoureux, E., Saw, S.M., Tay, W.T., Mitchell, P. and Wong, T.Y., 2010. Are myopic eyes less likely to have diabetic retinopathy?. Ophthalmology, 117(3), pp.524-530.

44. Baniasadi, N., Wang, M., Wang, H., Mahd, M. and Elze, T., 2017. Associations between optic nerve head-related anatomical parameters and refractive error over the full range of glaucoma severity. Translational vision science E technology, 6(4), pp.9-9. 
45. Wang, M., Jin, Q., Wang, H., Li, D., Baniasadi, N. and Elze, T., 2018. The interrelationship between refractive error, blood vessel anatomy, and glaucomatous visual field loss. Translational vision science $\mathcal{E}$ technology, 7(1), pp.4-4.

46. Hanruo Liu, Liu Li, I. Michael Wormstone, Chunyan Qiao, Chun Zhang, Ping Liu, Shuning Li, Huai zhou Wang, Dapeng Mou, Ruiqi Pang, Diya Yang, Lai Jiang, Yihan Chen, Man Hu, Yongli Xu, Hong Kang, Xin Ji, Robert Chang, Clement Tham, Carol Cheung, Daniel ShuWei Ting, Tien Yin Wong, Zulin Wang, Robert N. Weinreb, Mai Xu, Ningli Wang, Development and Validation of a Deep Learning System to Detect Glaucomatous Optic Neuropathy Using Fundus Photographs, JAMA Ophthalmology, 2019, pp. 1-8. 